\title{
IMPROVED SELF-SEALING LIQUID ENCAPSULATION IN PARYLENE STRUCTURES BY INTEGRATED STACKABLE ANNULAR-PLATE STICTION VALVE
}

Christian A. Gutierrez and Ellis Meng University of Southern California, Los Angeles, California, USA

\begin{abstract}
We report on an improved self-sealing structure for Parylene-based liquid encapsulation. The specific improvements are an integrated stiction valve which reduces overall device footprint by nearly $50 \%$ over inplane designs and the use of an annular-plate design in a stackable multi-layered configuration for successful longterm liquid encapsulation. We achieve automatic waferlevel liquid entrapment without using adhesives or processing at elevated pressures or temperatures. We also demonstrate the use of electrochemical impedance measurements as a means for tracking internal liquid volume.
\end{abstract}

\section{INTRODUCTION}

Many interesting MEMS applications such as variablefocus liquid lens optics, electrolysis actuators, and electrowetting devices employ encapsulated liquids [1]. These applications require appropriate fabrication techniques and structures for long term encapsulation of small liquid volumes. Parylene-based stiction valves have demonstrated excellent performance in wafer-level sealing of liquids within microchambers [2, 3], but are currently placed externally in relation to the liquid chamber and active structures of the device. This configuration unnecessarily increases the overall device footprint. Integration of the stiction valve within the active structure itself was attempted by [4] but liquid retention was limited to several minutes due to suboptimal valve design. Here we combine the optimal annular-plate valve design guidelines derived by [2] in an integrated device to reduce overall footprint by nearly $50 \%$ and achieve automatic long-term liquid encapsulation.

\section{DESIGN}

\section{Overall device design}

The device consists of three main components. 1) an annular-plate stiction valve, 2) peripheral fluidic access ports, and 3) a microchamber. The valve is connected via short microchannels to the fluidic access ports located on the periphery and base of the microchamber structure (Figure 1). The chamber is filled by passive soaking at the wafer level in the desired filling solution. Removal from the bath and exposure to ambient conditions induces evaporation through the peripheral access ports. The evaporating liquid fronts move along the connecting channels toward the stiction valve. This results in the stiction-induced collapse of the integrated annular-plate, thereby creating a uniform seal against the substrate trapping the remaining liquid in the chamber above the valve (Figure 2).

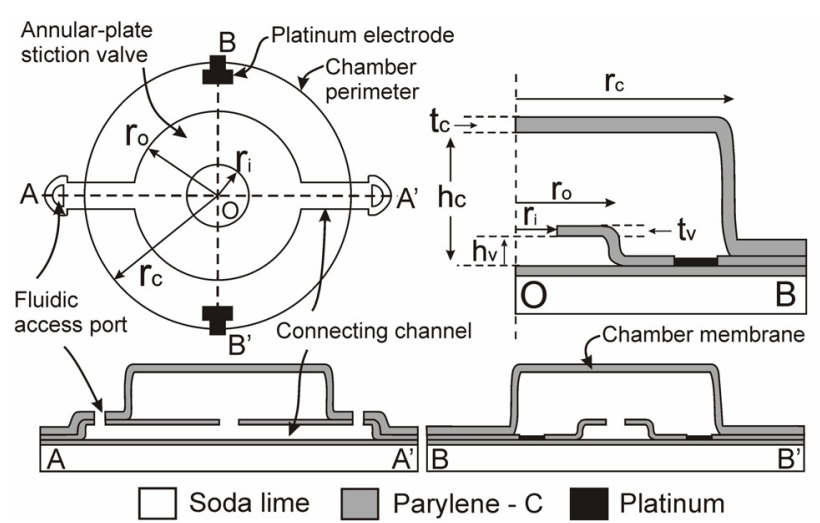

Figure 1 - Layout and key parameters. Top left: Top-view indicating key radii. Top right: Partial cross-section indicating key heights and thicknesses. Bottom row: Full cross-section images taken through the structure.

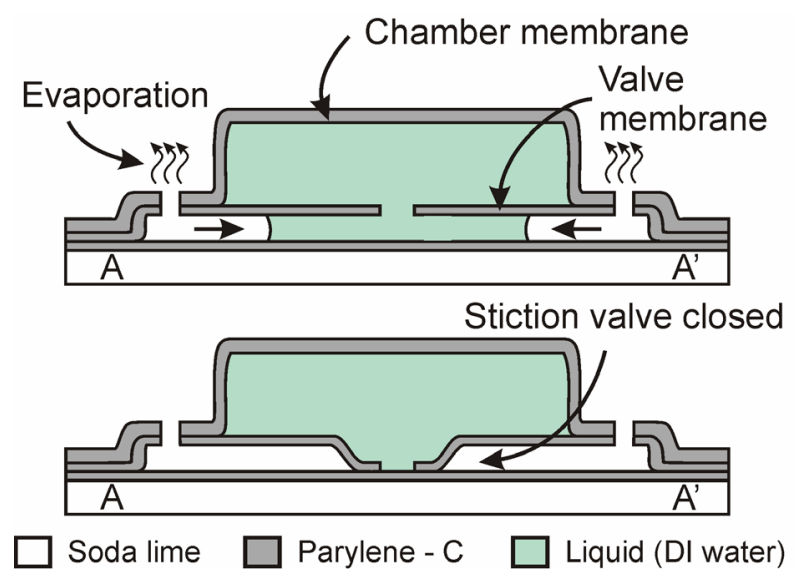

Figure 2 - Principle of operation. Evaporation through the fluidic access ports moves the liquid fronts along the connecting channels toward the stiction valve. Capillary forces seal the annular plate of the stiction valve against the substrate trapping liquid inside the chamber.

\section{Integrated stiction valve design guidelines}

The design guidelines for Parylene-based annular stiction valves were previously investigated $[2,5]$. Stiction occurs when the critical number $\left(N_{c}\right)$ exceeds 1 :

$$
N_{c}=\frac{\sigma r_{o}^{4}}{D h^{2}} p(\phi, v)
$$

where $\sigma=2 \gamma_{L A} \cos \theta_{C}$ is the surface energy of the Paryleneliquid interface, $D=\left(E t^{3} / 12\left(1-v^{2}\right)\right)$ is the flexural rigidity of the plate, $\gamma_{L A}$ is the liquid-air surface tension, $\theta_{C}$ is the Parylene contact angle, $E$ is Young's modulus, $t$ is the membrane thickness, $h$ is the membrane height, $v$ is Poisson's ratio, $\phi$ is the ratio $r_{i} / r_{o}$, and $p$ is a numerical function of $\phi$ and $v$ and has been evaluated through the 
use of numerical methods. A combination of parameters that satisfy the design constraints were selected for the device (Table 1). $N_{c}$ for these valve dimensions is well above 1 , ensuring that stiction occurs.

Table 1. Parameters selected for device fabrication

\begin{tabular}{|c|c|}
\hline Parameter & Dimension $(\mu \mathrm{m})$ \\
\hline$r_{o}$ & $63-100$ \\
\hline$r_{i}$ & $20-35$ \\
\hline$r_{c}$ & 150 \\
\hline$h_{v}$ & 2 \\
\hline$h_{c}$ & 12 \\
\hline$t_{v}$ & 2 \\
\hline$t_{c}$ & 4.2 \\
\hline
\end{tabular}

\section{FABRICATION}

The entire fabrication process is realized at relatively low temperatures $\left(90^{\circ} \mathrm{C}\right)$ which enables its use on a variety of polymer-based substrates and facilitates adoption in temperature sensitive processes. Standard soda-lime glass wafers were utilized as the substrate. Fabrication (Figure 3) began with optional platinum electrodes patterned on a $10 \mu \mathrm{m}$ Parylene film followed by a $1 \mu \mathrm{m}$ Parylene insulation layer (Fig. 3a, b). The $10 \mu \mathrm{m}$ Parylene film allows for release of structures and devices from the sodalime substrate for applications where flexibility is required. The optional electrodes are exposed to the liquid within the chamber and serve as an electrical interface for electrochemical measurement or actuation applications.

a
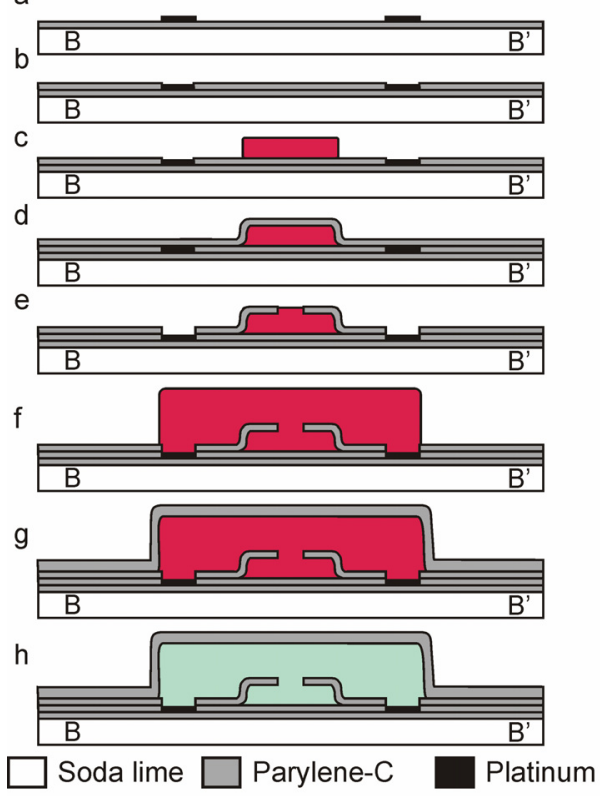

Photoresist $\square$ Liquid (DI water)

Figure 3 - Fabrication process shown through B-B' crosssection designated in Fig. 1. Standard surface micromachining techniques were utilized in this low temperature $\left(90^{\circ} \mathrm{C}\right)$ process enabling fabrication on a variety of polymer substrates.

$2 \mu \mathrm{m}$ of photoresist was then spun-on and patterned and $2 \mu \mathrm{m}$ of Parylene was deposited forming the valve height $\left(h_{v}\right)$, valve radius $\left(r_{o}\right)$, and valve membrane thickness $\left(t_{v}\right)$, respectively (Fig. 3c) . Parylene over the valve center and access ports was removed in oxygen plasma (100W, $100 \mathrm{mT}$ ) using a photoresist mask, thereby completing the annular valve structure $\left(r_{i}\right)$ and access ports (Fig. $3 \mathrm{~d}$, e).

The multi-layered valve-in-chamber sacrificial photoresist structure and the exposed state of the valve layer from the previous etching step made it difficult to remove the photoresist etch mask without erosion of the underlying valve structure. For this reason, the chamber sacrificial layer was spun and patterned directly on top of the etch mask (total of $12 \mu \mathrm{m}$ of photoresist) (Fig. 3f), ( $r_{c}$ $=150 \mu \mathrm{m})$. A $4.2 \mu \mathrm{m}$ Parylene film was then deposited $\left(t_{c}\right)$ to enclose the chamber and a final Parylene etching step re-opened the fluidic access ports (Fig. 3g). Following sacrificial photoresist removal in acetone, IPA, and DI water (Fig. 3h), the chambers were filled in a batch process by immersion in the desired fluid (DI water).

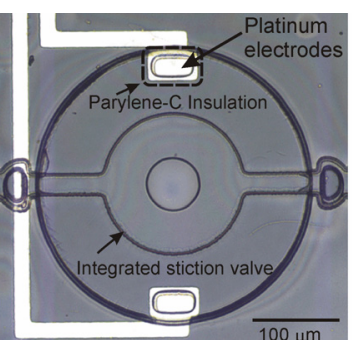

(a)

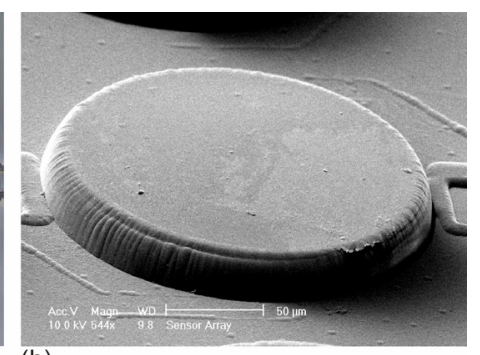

(b)
Figure 4 - (a) Fabricated device with optional electrodes; shown while still immersed in DI water. (b) SEM of fabricated chamber structure. A thin gold film was used to prevent charging under SEM observation.

\section{EXPERIMENTS AND RESULTS}

\section{Stiction valve sealing}

Stiction valve sealing and liquid encapsulation capability were evaluated by using a sample die containing several identical devices under optical observation. The devices were filled, as described previously, with DI water. The die was then removed from the bath and exposed to ambient laboratory conditions to initiate fluid encapsulation (Figure 5).

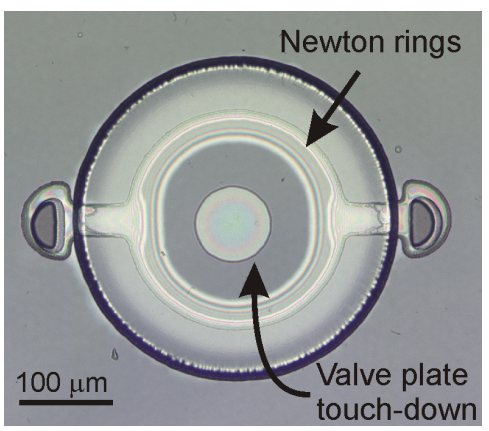

Figure 5 - Optical micrograph of $300 \mu$ m diameter device filled with DI water. The chamber is free standing while the valve is collapsed and pinned to substrate. The dark central region indicates the seal between the annularplate and substrate while interference rings indicate proximity to substrate as plate transitions from contact at the center to freestanding at the anchored edges. 


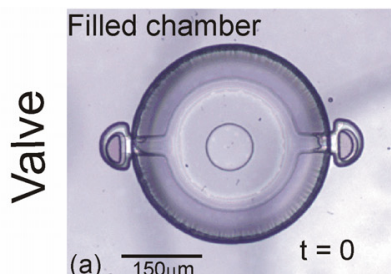

(a) $\overline{150 \mu \mathrm{m}}$

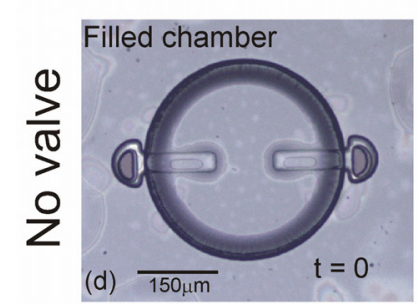

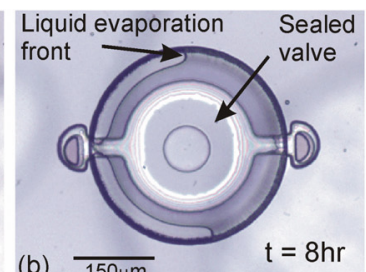

(b) $\overline{150 \mu \mathrm{m}}$

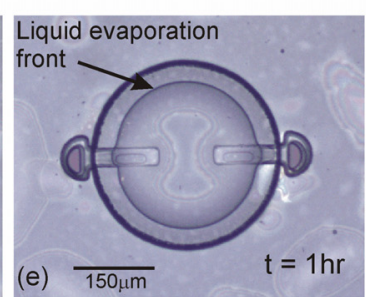

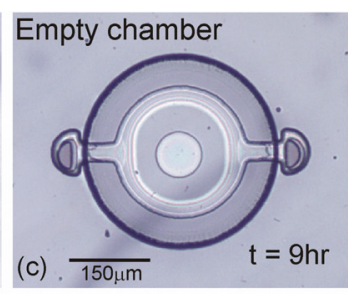

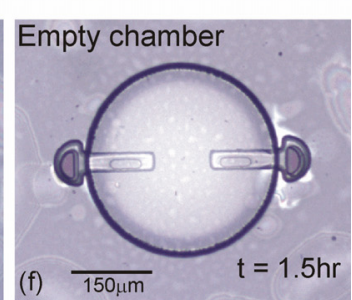

Figure 6 - (a) Completely filled chamber with valve immediately after removal from immersion bath. (b) Valved chamber after 8 hrs of evaporative drying at ambient conditions. Complete valve seal evidenced by interference rings and darker central annular region. (c) Empty chamber at 9hrs. Valve remains pinned to the substrate. (d) Chamber without valve immediately after removal from immersion bath. (e) Chamber drying occurs rapidly; liquid evaporation front appears at $1 \mathrm{hr}$. (f) At $1.5 \mathrm{hrs}$, the chamber is completely dry indicating rapid evaporation without a stiction valve.

Successful stiction valve sealing was clearly evidenced by the appearance of a dark central region indicating conformal contact between the annular plate and substrate. Emergence of interference rings between the central region and the peripheral plate anchors indicated proximity to the substrate as the valve plate transitioned from contact with the substrate to freestanding near the anchors.

\section{Liquid encapsulation}

Successful encapsulation of liquid was evidenced by the appearance of a liquid front within the chamber after several hours of complete encapsulation. Initially, the chambers appeared uniform due to complete filling and entrapment of liquid within the chamber (Figure 6a). This was followed by the appearance of a liquid front several hours later as a result of water vapor transmission (Figure 6b). Uniformly complete device filling is a unique feature not demonstrated previously; instead air pockets were often observed and thus, liquid entrapment was incomplete [4, 5]. For comparison, devices without a valve were also fabricated and tested (Figure 6d-f). Within 1.5 hours, valveless devices were completely dry while devices with a valve successfully encapsulated liquid up to 9 hours at ambient conditions $\left(20^{\circ} \mathrm{C}, \sim 30 \% \mathrm{RH}\right)$. The formation of a liquid front, even in valved devices, clearly indicated that liquid loss still occurred. Water vapor transmission through the chamber membrane and not the valve seal was hypothesized to be the root mechanism of liquid loss.

\section{Liquid loss mechanism}

In order to verify that water vapor transmission through the chamber membrane was the primary liquid loss mechanism, four devices were monitored optically under a microscope and electrochemically by solution impedance measurements via the integrated microelectrodes. Electrochemical impedance of the encapsulated liquid was measured $\left(5 \mathrm{kHz}, 100 \mathrm{mV}_{\mathrm{pp}}\right)$ over time using an Agilent precision LCR meter.

The impedance magnitude is a complex function of the ionic conductive path between the electrodes which varies over time as the chamber deforms to accommodate liquid volume loss. The phase angle is representative of liquid volume in the chamber by tracking the contribution of solution resistance to the measured phase. Impedance measurements were consistent across multiple devices and clearly tracked liquid volume changes over time. By correlating time stamped optical micrographs with measured impedance data, liquid loss was quantitatively tracked and measured (Figure 7). Phase angle measurements clearly followed a linear relationship with volume. As liquid was lost, the phase angle approached $-90^{\circ}$, indicating the transition from a mostly resistive phase angle contribution (i.e. solution present) to capacitive (i.e. no solution present).
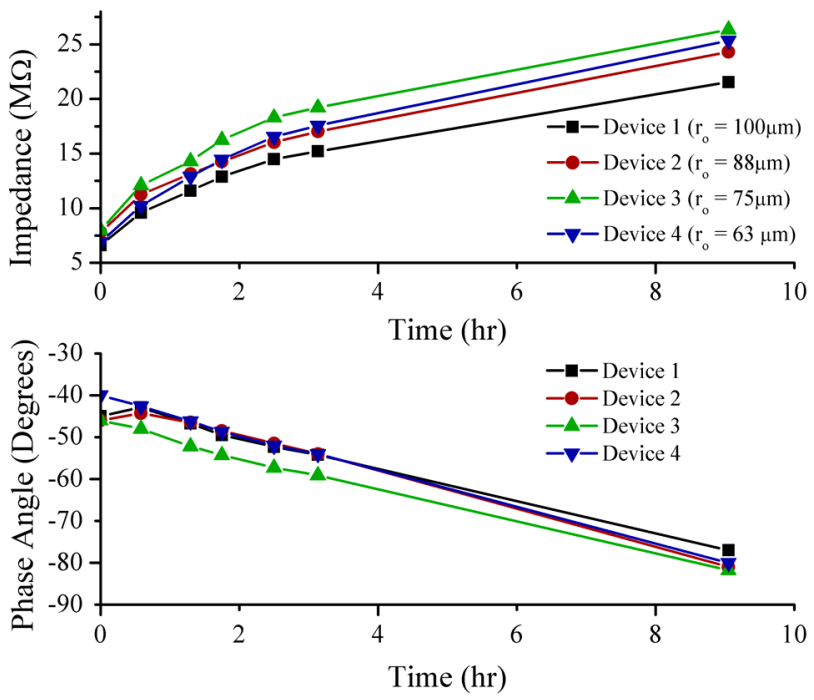

Figure 7 - Top: Impedance magnitude measured over time. Bottom: Phase angle approached $-90^{\circ}$ indicating a switch from resistive solution impedance (wet chamber) to capacitive impedance (dry chamber).

The change in liquid volume over time (Figure 8) corresponds to a measured water vapor transmission rate 
(WVTR) of $5.6 \times 10^{-12} \mathrm{~g} \cdot \mu \mathrm{m} / \mu \mathrm{m}^{2} \cdot \mathrm{hr}$ through top surface of the chamber. This value agrees closely with the previously reported WVTR value of $5.78 \times 10^{-12} \mathrm{~g} \cdot \mu \mathrm{m} / \mu \mathrm{m}^{2} \cdot \mathrm{hr}\left(20^{\circ} \mathrm{C}\right.$, $30 \% \mathrm{RH}$ ) for Parylene $\mathrm{C}$ films [6]. This result confirms that the liquid loss mechanism is indeed water vapor transmission directly through the Parylene membrane and not the stiction valve seal. Electrochemical impedance measurements provide a simple and reliable method to quantitatively track internal liquid volumes over time.

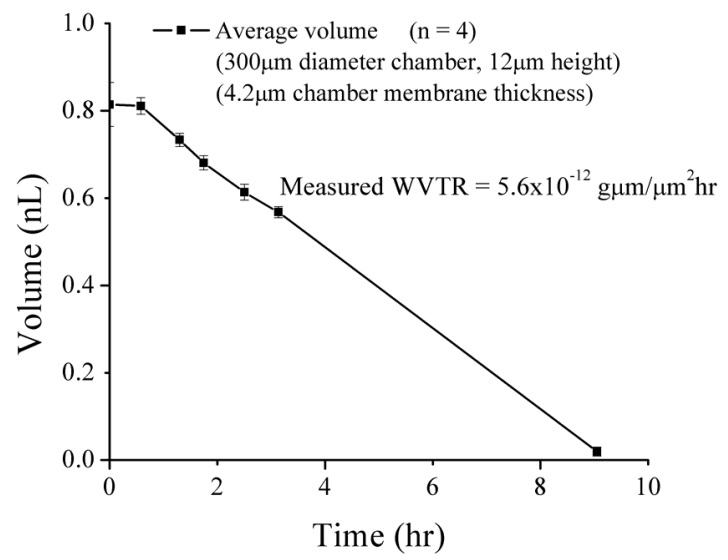

Figure 8 - Liquid volume measured over time (mean $\pm S D$, $n=4)$. The measured WVTR agreed closely with previously reported values for Parylene films at ambient conditions. This indicated that the duration of liquid encapsulation was primarily limited by the water vapor transmission of the Parylene film and not valve sealing. Volume was tracked optically and measured using phase angle.

\section{DISCUSSION AND APPLICATIONS}

The performance-limiting factor for liquid encapsulation duration is the water vapor transmission rate through asdeposited Parylene films. To further improve performance, additional techniques can be implemented to enhance encapsulation lifetime $\left(h_{\text {encapsulation }}\right)$. The parameters affecting $h_{\text {encapsulation }}$ are identified below:

$$
h_{\text {encapsulation }}=\frac{M t}{W V T R \cdot S A}
$$

where $M$ is mass of water (grams), $t$ is thickness of the Parylene membrane $(\mu \mathrm{m})$, and $S A$ is exposed surface area available for transmission in $\left(\mu \mathrm{m}^{2}\right)$. Increasing membrane thickness improves encapsulation lifetime proportionally while WVTR and exposed surface area are inversely proportional. The WVTR of as-deposited Parylene-C films can be reduced by nearly $50 \%$ with additional high temperature annealing steps $[4,6]$. Furthermore, deposition of thin films such as gold or aluminum can also be used to reduce the SA available for transmission and provide a nearly impermeable protective coating [5]. The use of a combination of these techniques can feasibly extend encapsulation time for sub-nL volumes (in the current design) out to several days.

The valve-in-chamber configuration is useful for applications in electrochemical-based transducers such as force and pressure sensors. The capability of small encapsulated liquid volumes to measure contact forces and hydrostatic pressures has been demonstrated with excellent sensitivity [7]. Furthermore, electrolysis-based actuation can be fully exploited by this design as increasing chamber pressure further enforces the seal between the valve plate and substrate in contrast to previously reported in-plane designs in which the valve opened at sufficiently high internal pressures[3, 5].

\section{CONCLUSION}

The technique utilized here improves encapsulation performance by integrating an optimized stiction valve within a chamber structure. This reduces overall device footprint by nearly $50 \%$ over in-plane approaches, extends encapsulation lifetime, is performed in batch at the waferlevel, involves no high temperature or pressure steps and is achieved without adhesives. This is accomplished at the cost of only a few additional standard micromachining processing steps. This improved approach has the potential to enhance many applications where liquid encapsulation is required.

\section{ACKNOWLEDGEMENTS}

This work was funded in part by the Engineering Research Centers Program of the NSF under Award Number EEC-0310723 and the Bill and Melinda Gates Foundation (CG). The authors would like to thank Dr. Donghai Zhu and the members of the USC Biomedical Microsystems Laboratory for their assistance.

\section{REFERENCES}

[1] J. Lee and C.-J. Kim, "Surface-tension-driven microactuation based on continuous electrowetting," Microelectromechanical Systems, Journal of, vol. 9, pp. 171-180, 2000.

[2] Z. Wang and $\mathrm{Y}$. $\mathrm{Xu}$, "Theoretical and Experimental Study of Annular-Plate SelfSealing Structures," Microelectromechanical Systems, Journal of, vol. 17, pp. 185-192, 2008.

[3] C. A. Gutierrez and E. Meng, "A Dual Function Parylene-based Biomimetic Tactile Sensor and Actuator for Next Generation Mechanically Responsive Microelectrode Arrays," in Proc. Transducers 2009, Denver, CO, USA, pp. 21942197.

[4] S. Matsumoto and N. Ichikawa, "New Methods for Liquid Encapsulation in Polymer MEMS Structures," in Proc. MEMS 2008, Tucson, AZ, USA, pp. 415-418.

[5] H. Zhang, S. Wang, et al., "Study and Applications of a Parylene Self-Sealing Structure," in Proc. MEMS 2006, pp. 282-285.

[6] P. R. Menon, W. Li, et al., "Characterization of Water Vapor Permeation Through Thin Film Parylene C," in Proc. Transducers 2009, Denver, CO, USA, pp. 1892-1895.

[7] D. A. Ateya, A. A. Shah, et al., "Impedancebased response of an electrolytic gas bubble to pressure in microfluidic channels," Sensors and Actuators A, vol. 122, pp. 235-241, 2005. 